\title{
Vitamin D status is associated with cardiometabolic markers in 8-11-year-old children, independently of body fat and physical activity
}

\author{
Rikke A. Petersen ${ }^{1,2}$, Stine-Mathilde Dalskov ${ }^{1}$, Louise B. Sørensen ${ }^{1}$, Mads F. Hjorth ${ }^{1}$, Rikke Andersen ${ }^{3}$, \\ Inge Tetens ${ }^{3}$, Henrik Krarup ${ }^{4}$, Christian Ritz ${ }^{1}$, Arne Astrup ${ }^{1}$, Kim F. Michaelsen ${ }^{1}$, Christian Mølgaard ${ }^{1}$ \\ and Camilla T. Damsgaard ${ }^{1_{*}}$ \\ ${ }^{1}$ Department of Nutrition, Exercise and Sports, Faculty of Science, University of Copenhagen, Denmark, Rolighedsvej 26, \\ 1958 Frederiksberg C, Denmark \\ ${ }^{2}$ University College Lillebaelt, Blangstedgårdsvej 4, 5220 Odense SØ, Denmark \\ ${ }^{3}$ Division of Nutrition, The National Food Institute, Technical University of Denmark, Mørkhøj Bygade 19, 2860 Søborg, \\ Denmark \\ ${ }^{4}$ Department of Clinical Biochemistry, Section of Molecular Diagnostics, Aalborg University Hospital, Hobrovej 18-22, \\ 9000 Aalborg, Denmark
}

(Submitted 17 March 2015 - Final revision received 9 July 2015 - Accepted 16 July 2015 - First published online 18 September 2015)

\section{Abstract}

Vitamin D status has been associated with cardiometabolic markers even in children, but the associations may be confounded by fat mass and physical activity behaviour. This study investigated associations between vitamin D status and cardiometabolic risk profile, as well as the impact of fat mass and physical activity in Danish 8-11-year-old children, using baseline data from 782 children participating in the Optimal well-being, development and health for Danish children through a healthy New Nordic Diet (OPUS) School Meal Study. We assessed vitamin D status as serum 25-hydroxyvitamin D (25(OH)D) and measured blood pressure, fasting plasma glucose, homoeostasis model of assessment-insulin resistance, plasma lipids, inflammatory markers, anthropometry and fat mass by dual-energy X-ray absorptiometry, and physical activity by $7 \mathrm{~d}$ accelerometry during August-November. Mean serum 25(OH)D was $60 \cdot 8(\mathrm{sD} 18 \cdot 7) \mathrm{nmol} / \mathrm{l}$. Each $10 \mathrm{mmol} / \mathrm{l} 25(\mathrm{OH}) \mathrm{D}$ increase was associated with lower diastolic blood pressure $(-0.3 \mathrm{mmHg}, 95 \% \mathrm{CI}-0.6,-0.0)(P=0.02)$, total cholesterol $(-0.07 \mathrm{mmol} / \mathrm{l}$, $95 \%$ CI $-0 \cdot 10,-0 \cdot 05)$, LDL-cholesterol $(-0.05 \mathrm{mmol} / 1,95 \% \mathrm{CI}-0 \cdot 08,-0 \cdot 03)$, TAG $(-0.02 \mathrm{mmol} / 1,95 \%$ CI $-0 \cdot 03,-0 \cdot 01)(P \leq 0 \cdot 001 \mathrm{for}$ all lipids) and lower metabolic syndrome (MetS) score $(P=0 \cdot 01)$. Adjustment for fat mass index did not change the associations, but the association with blood pressure became borderline significant after adjustment for physical activity $(P=0 \cdot 06)$. In conclusion, vitamin $\mathrm{D}$ status was negatively associated with blood pressure, plasma lipids and a MetS score in Danish school children with low prevalence of vitamin D deficiency, and apart from blood pressure the associations were independent of body fat and physical activity. The potential underlying cause-effect relationship and possible long-term implications should be investigated in randomised controlled trials.

Key words: Vitamin D: Cardiometabolic health: Children

Vitamin D status is assessed by the concentration of serum 25-hydroxyvitamin D $(25(\mathrm{OH}) \mathrm{D})$, a circulating vitamin D metabolite $^{(1)}$. Humans obtain vitamin D from exposure to sunlight and through vitamin $\mathrm{D}$ intake, with sunlight being the primary source ${ }^{(1)}$. Vitamin $\mathrm{D}$ facilitates intestinal $\mathrm{Ca}$ absorption $^{(2,3)}$, and severe deficiency is detrimental to childhood bone health ${ }^{(4)}$. In addition, vitamin D status in childhood has been inversely associated with cardiometabolic risk markers such as glucose, insulin and blood pressure ${ }^{(5)}$. These associations have been suggestively explained by a potential favourable role of active vitamin $\mathrm{D}, 1,25$-dihydroxyvitamin $\mathrm{D}\left(1,25(\mathrm{OH})_{2} \mathrm{D}\right)$, in cardiometabolic mechanisms such as blood pressure regulation through the renin-angiotensin system ${ }^{(6)}$, pancreatic $\beta$-cell function and protection ${ }^{(7,8)}$, and low-grade inflammation suppression ${ }^{(9,10)}$, as well as by an inverse association with parathyroid hormone (PTH), an apparent predictor of inflammation, dyslipidaemia and cardiovascular calcifications in obese children and adolescents ${ }^{(11,12)}$.

Excess body weight and fat mass in childhood increase the risk of the metabolic syndrome (MetS), a cluster of elevated risk factors including insulin resistance, high blood pressure, deranged lipid profile and abdominal obesity, that has been associated with CVD risk in adults ${ }^{(13,14)}$. BMI is consistently

Abbreviations: 25(OH)D, 25-hydroxyvitamin D; FMI, fat mass index; HOMA-IR, homoeostasis model of assessment of insulin resistance; MetS, metabolic syndrome; MVPA, moderate-to-vigorous physical activity; PTH, parathyroid hormone.

* Corresponding author: Professor C. T. Damsgaard, fax 453533 2483, email ctd@nexs.ku.dk 
inversely associated with vitamin D status in adults ${ }^{(15)}$. This association has also been demonstrated in overweight and obese children $^{(16-18)}$, and in American and Spanish children of primarily normal weight ${ }^{(19-22)}$, although not all studies agree $^{(23,24)}$. Relations between vitamin D status and cardiometabolic risk markers may be mediated or confounded by fat mass, which in turn may be associated with vitamin D status through sequestering of vitamin $\mathrm{D}$ in adipose tissue $\mathrm{e}^{(25)}$ or by reverse causality - that is, through poorer diets and less sun exposure in overweight subjects leading to lower vitamin D status. Therefore, adjustment for the potential confounding effects of fat mass is important when evaluating associations between vitamin D status and cardiometabolic health. Most studies in children have used BMI, which does not convey information on body composition, and more sophisticated measurements of body fat are called for in observational studies $^{(25)}$. Moreover, as physical activity, especially of moderate-to-vigorous physical activity (MVPA), has been associated both with cardiometabolic risk markers ${ }^{(26)}$ and with increased sun exposure and vitamin D status in children ${ }^{(27)}$, it should be considered a potential confounder.

The aim of this cross-sectional study was to (1) investigate associations between vitamin D status and cardiometabolic risk markers, and (2) investigate whether these associations were independent of objectively measured fat mass index (FMI) and physical activity, in a large cohort of Danish 8-11-year-old school children.

\section{Methods}

\section{Study design and participants}

The present study used cross-sectional baseline data from the Optimal well-being, development and health for Danish children through a healthy New Nordic Diet (OPUS) School Meal Study, a large cluster-randomised controlled cross-over trial including third- and fourth-grade children from nine schools in Denmark. The primary aim of the OPUS School Meal Study was to evaluate the impact of school meals based on the New Nordic Diet on a MetS score and cognitive concentration performance, as previously described ${ }^{(28)}$. Briefly, initial contact was established with thirty-nine schools whereof nine schools, located in eight separate urban and rural municipalities, were included. Inclusion criteria for the schools were (a) location in the eastern part of Denmark; (b) a total of four or more classes at third and fourth grade; (c) suitable kitchen facilities available for food production during school days; and (d) a high motivation for participation as determined by the study team. Written information about the study was sent to the families of all 1021 third- and fourth-grade children at the schools. To recruit families of various socioeconomic and ethnic backgrounds, we held several information meetings at each school; sent out several reminders; and had translators present at meetings when relevant. Exclusion criteria for the children were (a) diseases or conditions that might obstruct the measurements or put a child at risk by eating the intervention school meals, or (b) concomitant participation in other studies that involved radiation or blood sampling. Written informed consent for participation was obtained from the custody holders of 834 children (82\%).
The study was conducted in accordance with the Declaration of Helsinki, and the study protocol was approved by the Committees on Biomedical Research Ethics for the Capital Region of Denmark (H-1-2010-124) and registered with clinicaltrials.gov (NCT 01577277).

\section{Questionnaires}

Information on demographic and socioeconomic characteristics was collected by an in-depth $2 \mathrm{~h}$ personal interview with the child and parents. On the basis of the highest education level attained in the household, parental education level was categorised as lower secondary education ( $\leq 10$ years of schooling), upper secondary education, vocational education, short higher education, bachelor's degree or equivalent or $\geq$ master's degree ( $\geq 17$ years of schooling). Children were categorised as immigrants/descendants if all grandparents and $\geq 1$ parent were born outside Denmark. This classification was inspired by the definitions of immigrants and descendants used by Statistics Denmark $^{(29)}$, although not identical as citizenship was not available in the present study. Pubertal status was self-evaluated by the child based on breast development in girls and pubic hair in boys (Tanner stages) ${ }^{(30)}$. Registrations were dichotomised into a variable indicating entered puberty (yes/no).

\section{Recordings of dietary intake and physical activity}

During the weeks before the clinical examination, children, aided by their parents, recorded their daily intake of food and beverages for 7 consecutive days using a Web-based Dietary Assessment Software for Children. The software was developed specifically for children aged 8-11 years and validated in the OPUS School Meal Pilot Study ${ }^{(31,32)}$. Intake data were processed as previously described ${ }^{(31)}$. Energy intake (EI) relative to estimated BMR was evaluated following the equations by Henry $^{(33)}$, and energy under-reporters $(\mathrm{EI} / \mathrm{BMR} \leq 1.05)$ and over-reporters $(\mathrm{EI} / \mathrm{BMR} \geq 2 \cdot 28)^{(34)}$ were excluded from the dietary variables, as were children who registered their diet for $<4 \mathrm{~d}$. Intake of dietary supplements was registered and a dichotomous variable was formed, indicating whether the child had ingested any kind of vitamin D-containing dietary supplements, including multivitamins, during the dietary registration week. To measure physical activity, the children were asked to wear an accelerometer (ActiGraph GT3X+ Tri-Axis Accelerometer Monitor) in an elastic belt tightly fastened at the right hip for the same $7 \mathrm{~d}$ and eight nights as the dietary recordings, and to remove it only during water activities - that is, showering or swimming. Analyses of the physical activity data were described in detail previously ${ }^{(35)}$. We used time spent with MVPA, defined as $\geq 2296$ vertical counts $/ \mathrm{min}^{(36)}$, as the measure of physical activity, as this has shown stronger associations with cardiometabolic risk markers ${ }^{(26)}$ than total physical activity (counts/min) in children.

\section{Clinical measurements and blood sampling}

Clinical measurements and blood sampling were conducted from 30 August to 28 November 2011 during morning hours at 
each school, as previously described ${ }^{(37)}$. Children had been fasting since midnight, had voided and were wearing only light clothing. Height was measured to the nearest one decimal using a transportable stadiometer (CMS Weighing Equipment Ltd). Height was derived as the mean of three consecutive measurements with the child in standing position and holding his head in the Frankfurter plane and body weight was measured using a digital scale (BWB $800 \mathrm{~S}$; Tanita). BMI was calculated as body weight $(\mathrm{kg})$ divided by height $\left(\mathrm{m}^{2}\right)$, and sex-adjusted and age-adjusted $Z$-scores for BMI were calculated using the WHO AnthroPlus software ${ }^{(38)}$. Children were categorised as underweight, normal weight, overweight and obese on the basis of age- and sex-specific cut-offs defined to pass through BMI of $18.5,25$ and $30 \mathrm{~kg} / \mathrm{m}^{2}$ at the age of 18 years, as described by Cole et $a l{ }^{(39,40)}$. Waist circumference was measured in centimetres to the nearest millimetre using a non-elastic measuring tape at the level of the umbilicus, and the mean of three consecutive measurements was used. Total body fat mass was determined by dual-energy X-ray absorptiometry (DXA) scanning (Lunar Prodigy Pro ${ }^{\mathrm{TM}}$; GM Healthcare) with EnCoreTM software. FMI was calculated as total fat mass divided by height squared. In addition, the investigator recorded the apparent ethnicity of the child by applying the terminology of the DXA software - that is, Caucasian, Asian, African, Latin or Other. Children from Turkey, the Middle East, Pakistan and India were recorded as Caucasians. Because of small categories, a dichotomised variable indicating whether or not the child was Caucasian (yes/no) was used.

Blood pressure and heart rate were measured by an automated device (UA-787 Plusl; A\&D Medical) after 10 min of rest using two different cuff sizes (18-22 or $22-32 \mathrm{~cm}$ ). A second device (ProBP 3400 Sure BP; Welch Allyn Inc.) was used for children with arm circumferences $<18 \mathrm{~cm}$. Measurements were performed three times whereof the mean of the last two measurements was used. Thereafter, fasting blood was sampled from the forearm by venepuncture. Local anaesthetic patches were offered to the children to reduce discomfort of blood sampling.

\section{Blood analyses}

Whole-blood glucose concentrations were analysed immediately after sampling (Glucose 201; Hemocue Danmark). Twenty-four glucose samples were removed from the analyses because they were obtained from non-fasting children. Heparinised blood and blood with EDTA were centrifuged at $2500 \boldsymbol{g}$ for $10 \mathrm{~min}$ at room temperature, and plasma was stored at $-80^{\circ} \mathrm{C}$ for batch analysis of total, LDL and HDL-cholesterol, TAG, IL- 6 and adiponectin. Blood collected in serum tubes with gel was centrifuged after $30 \mathrm{~min}$ of coagulation at room temperature, and it was stored at $-80^{\circ} \mathrm{C}$ for later batch analysis of insulin, 25(OH)D and intact PTH. For each analysis, all samples were ran on the same device with the same reagent lot, all samples from each child were analysed on the same day and all samples from one school were analysed in one assay. Cholesterols and TAG were quantified on the Vitros 5.1 FS analyser (Ortho-Clinical Diagnostics, Johnson \& Johnson). LDL-cholesterol was calculated using Friedewalds's equation $^{(41)}$. Plasma high-sensitivity IL-6 and adiponectin were measured in duplicate by ELISA (R\&D Systems Europe Ltd), and serum insulin was quantified on ADVIA Centaur XP (Siemens Healthcare). Homoeostasis model of assessment of insulin resistance (HOMA-IR) was calculated as plasma glucose $(\mathrm{mmol} / \mathrm{l}) \times$ serum insulin $(\mathrm{mIU} / \mathrm{l}) / 22 \cdot 5^{(42)}$, with insulin concentrations converted from $\mathrm{pmol} / \mathrm{l}$ to $\mathrm{mIU} / \mathrm{l}$ by dividing by 6.945. Twenty-three insulin and HOMA-IR samples were removed from the analyses because they were obtained from non-fasting children.

Vitamin D status was measured as serum 25(OH)D concentrations (including both $\mathrm{D}_{2}$ and $\mathrm{D}_{3}$ ) by an automated chemiluminescent immunoassay on DiaSorin LIAISON (DiaSorin $\mathrm{AB}$ ) at the Department of Clinical Biochemistry, Aalborg University Hospital, Aalborg, Denmark, a laboratory partaking in the vitamin D external quality assessment scheme (DEQAS) ${ }^{(43)}$. During the serum $25(\mathrm{OH}) \mathrm{D}$ analysis period (May to September 2012), the DEQAS quality control showed that our analysis had a negative bias of up to $30.6 \%$ compared with the all laboratory trimmed means (ALTM) and a bias of $2.3-14.5 \%$ compared with the means of laboratories using the same method as we used (method mean). Accordingly, the results of the laboratory are always within 2 SD but mostly within 1 SD of ALTM with a tendency to be slightly lower. Before analysis, serum was thawed at room temperature for $30 \mathrm{~min}$ before being centrifuged at $4000 \mathrm{rpm}$ for $3 \mathrm{~min}$. Concentrations are presented in $\mathrm{nmol} / \mathrm{l}(1 \mathrm{nmol} / \mathrm{l}=0.4006 \mathrm{ng} / \mathrm{ml})$, and lowest detection limit was $10 \mathrm{nmol} / \mathrm{l}$. Vitamin D deficiency and insufficiency was defined as serum 25(OH)D concentrations $<25$ and $\leq 50 \mathrm{nmol} / \mathrm{l}$, as recommended by the European Society for Paediatric Gastroenterology, Hepatology and Nutrition ${ }^{(44)}$

Serum intact PTH concentrations were determined by a chemiluminescent immunoassay on ADVIA Centaur XP (Siemens Healthcare). Lowest detection limit was $0.265 \mathrm{pmol} / \mathrm{l}$. One sample had PTH concentration below the detection limit of $0.265 \mathrm{pmol} / \mathrm{l}$; this sample was defined as half of the detection limit $(0 \cdot 133 \mathrm{pmol} / \mathrm{l})$, and one doubtful PTH value of $109 \mathrm{pmol} / 1$ $(25(\mathrm{OH}) \mathrm{D}$ at $89 \cdot 1 \mathrm{nmol} / \mathrm{l})$ was removed from the data set as extreme outlier.

EPA + DHA in whole blood, a biomarker of fish and $n-3$ long-chain PUFA intake, was measured by high-throughput GC within 3 months after blood sampling, as previously described ${ }^{(45)}$. The content of individual fatty acids and fatty acid classes are given in weight $\%$ of the total whole-blood fatty acids.

The inter- and intra-assay CV for the blood analyses were as follows: 1.4 and $1.2 \%$ (total cholesterol); 2.0 and $1.2 \%$ (HDLcholesterol); 1.5 and $0.8 \%$ (TAG); 6.7 and 2.9\% (IL-6); 11 and $3.8 \%$ (adiponectin); 2.5 and $3.1 \%$ (insulin); 5.4 and $7.6 \%$ $(25(\mathrm{OH}) \mathrm{D}) ; 7.4$ and $7.9 \%(\mathrm{PTH}) ; 4.5$ and $1.3 \%(\mathrm{EPA})$; and 6.4 and $2.4 \%$ (DHA). The inter-assay variation for glucose was $4.0 \%$.

\section{Metabolic syndrome score and clinically high values}

A continuous MetS score was calculated by combining the $Z$-scores derived from separate analyses of the five outcomes: mean arterial pressure, plasma HDL-cholesterol, plasma TAG, HOMA-IR and waist circumference. This was based on the 
International Diabetes Federation (IDF) definition of the MetS for children $\geq 10$ years of age ${ }^{(13)}$, although we included HOMA-IR rather than blood glucose because the latter is highly stable in non-diabetic children, even among obese adolescents with MetS features ${ }^{(46)}$. In addition, the cut-offs recommended by the IDF for diagnosing the MetS in $\geq 10$-year-olds were used in the present study to identify clinically aberrant values of the individual MetS markers ${ }^{(13)}$. These were $\geq 130$ and $\geq 85$ for systolic and diastolic blood pressure, respectively; $\geq 1.7$ and $<1.03 \mathrm{mmol} / 1$ for TAG and HDL-cholesterol, respectively; and $\geq 90$ th percentile for waist circumference, using a recently published Danish reference material for waist circumference in children $^{(47)}$. No widely used cut-offs for HOMA-IR have been defined in children. The cut-off of 2.5 used in adults has been applied, but as it has been argued that it should be closer to 3 in growing children of this age ${ }^{(48)}$ the cut-off for HOMA-IR was set at $>2.8$ in the present study. For total and LDL-cholesterol, values $\geq 200 \mathrm{mg} / \mathrm{dl}(\geq 5.2 \mathrm{mmol} / \mathrm{l})$ and $\geq 130 \mathrm{mg} / \mathrm{dl}(\geq 3.4 \mathrm{mmol} / \mathrm{l})$ were defined as elevated, as recommended by the American Academy of Pediatrics ${ }^{(49)}$

\section{Statistical analyses}

The statistical analyses were carried out using STATA (version 12.1; StataCorp). Assumptions underlying statistical models were assessed graphically using residual and normal probability plots, and outcomes were logarithm-transformed as appropriate. Significance was established at $P<0 \cdot 05$. Descriptive characteristics of the study sample are presented as means and standard deviations, medians (25th-75th percentile) or proportions (\%), and sex differences in continuous variables were tested using the two-sample $t$ test or Wilcoxon-MannWhitney test (non-normally distributed variables), and with the $\chi^{2}$ test or Fisher's exact test for categorical variables.

Linear mixed-effects models were used to evaluate associations between serum 25(OH)D and the cardiometabolic markers, and in all models school, grade, class and sibling were included as random effects to account for the dependency caused by the nested design. Sex, age, height, ethnicity, entered puberty (yes/no) and parental education were included as fixed effects, as well as EPA+DHA in whole blood, which has previously been associated with cardiometabolic health in the current study population ${ }^{(45)}$. In addition, an interaction term between sex and puberty was included in the models, but it was removed if nonsignificant. In addition, all associations including blood pressure were adjusted for the blood pressure device applied.

Additional adjustment for FMI (or BMI-for-age $Z$-scores), MVPA, as well as PTH, as potential confounders or mediators were performed subsequently in those models that had initially shown significant associations between $25(\mathrm{OH}) \mathrm{D}$ and cardiometabolic markers, by including one covariate at a time. High colinearity meant that waist circumference and the MetS score were not adjusted for FMI or BMI $Z$-scores. All estimated regression coefficients are presented as outcome response per $10 \mathrm{nmol} / 1$ increase in serum 25(OH)D.

Finally, we explored whether serum $25(\mathrm{OH}) \mathrm{D}$ was associated with elevated cardiometabolic values, using logistic regression analysis. These models included the same fixed effects as the linear regression models described above. In case of convergence problems, reduced logistic regression models were fitted including only class as random effect or no random effects.

\section{Results}

\section{Characteristics of the study population}

Of the 834 children originally enroled in the OPUS School Meal Study, eleven children withdrew before the first blood sampling, seven were ill or on holiday on the day of sampling and thirty-four children failed to deliver a blood sample or had a sample with insufficient blood volume for 25(OH)D analysis. Accordingly, 782 children (93.8\%) were included in the present study. There were 47.7 girls and $52.3 \%$ boys and most children $(94.7 \%)$ were of Caucasian ethnicity, whereas 2.6, 1.8, 0.8 and $0 \cdot 1 \%$ were of Asian, Afro-American, Latin-American and other ethnicities, respectively. As will be published elsewhere (Rikke A. Petersen, unpublished results), the serum 25(OH)D concentrations ranged from 15 to $132 \mathrm{nmol} / 1$ and concentrations $\leq 50 \mathrm{nmol} / 1$ were observed in $28.4 \%$ of the children, whereas $2.4 \%$ had concentrations $<25 \mathrm{nmol} / \mathrm{l}$. About half of the children had taken vitamin D-containing supplements during the dietary registration week. Boys were older, spent more time on MVPA, had higher intakes of energy, protein, vitamin D and $\mathrm{Ca}$ and higher serum 25(OH)D than girls, whereas more girls had entered puberty (Table 1). In addition, girls had higher FMI and higher values of most cardiometabolic markers compared with boys (Table 2).

\section{Associations between vitamin D status and BMI, fat mass} index, physical activity and parathyroid hormone

Serum 25(OH)D was negatively associated with BMI $Z$-scores $(P=0.03)$ and FMI $(P=0.001)$ and positively associated with MVPA $(1.21 \mathrm{~min} / \mathrm{d}, 95 \%$ CI $0 \cdot 29,2 \cdot 12 / 10 \mathrm{nmol} / 1$ increase) $(P=0 \cdot 010)$. However, the association with BMI $Z$-scores became nonsignificant when the model was adjusted for parental education $(-0.03,95 \% \mathrm{CI}-0.07,-0 \cdot 001, P=0.14)$. In contrast, the negative association between serum $25(\mathrm{OH}) \mathrm{D}$ and FMI $\left(-0.09 \mathrm{~kg} / \mathrm{m}^{2}, 95 \% \mathrm{CI}-0 \cdot 16,-0.02, P=0.007\right)$, as well as the positive association with MVPA ( $1.29 \mathrm{~min} / \mathrm{d}$ (95\% CI $0 \cdot 36,2 \cdot 23, P=0 \cdot 007)$, remained after adjustment for parental education. Moreover, serum $25(\mathrm{OH}) \mathrm{D}$ was negatively associated with PTH, corresponding to $0.23 \mathrm{pmol} / 1$ (95\% CI $0 \cdot 16$, $0 \cdot 29, P<0 \cdot 001)$ lower PTH per $10 \mathrm{nmol} / 1$ increase in $25(\mathrm{OH}) \mathrm{D}$.

\section{Associations between vitamin D status and cardiometabolic} risk markers

Serum 25(OH)D was negatively associated with diastolic blood pressure, total and LDL-cholesterol, TAG and the MetS score, but not with HOMA-IR, systolic blood pressure, HDL-cholesterol or waist circumference (Table 3). Adjustment for FMI did not change any of the associations, whereas the association between serum 25(OH)D and diastolic blood 
Table 1. Characteristics of the study population

(Mean values and standard deviations; median values and 25th, 75th percentiles; percentages)

\begin{tabular}{|c|c|c|c|c|c|}
\hline & \multicolumn{2}{|c|}{ Girls } & \multicolumn{2}{|c|}{ Boys } & \multirow[b]{2}{*}{$P$} \\
\hline & Mean & SD & Mean & SD & \\
\hline Age (years) & $9 \cdot 9$ & $0.6^{*}$ & $10 \cdot 1$ & 0.6 & $<0.001$ \\
\hline Entered puberty (\%) & \multicolumn{2}{|c|}{46.6} & \multicolumn{2}{|c|}{$23 \cdot 7$} & $<0.001$ \\
\hline Non-Caucasian (\%) & \multirow{2}{*}{\multicolumn{2}{|c|}{$\begin{array}{r}4 \cdot 8 \\
10 \cdot 7\end{array}$}} & \multirow{2}{*}{\multicolumn{2}{|c|}{$5 \cdot 7$}} & 0.61 \\
\hline Immigrant/descendants (\%) & & & & $12 \cdot 2$ & 0.51 \\
\hline Parental education (\% A/B/C/D/E/F) & \multicolumn{2}{|c|}{ 6/4/33/10/28/19 } & \multicolumn{2}{|c|}{$5 / 3 / 31 / 10 / 28 / 23$} & 0.75 \\
\hline $\begin{array}{l}\text { BMl-for-age Z-score } \\
\text { FMl }\left(\mathrm{kg} / \mathrm{m}^{2}\right)\end{array}$ & 0.08 & 1.04 & $0 \cdot 21$ & $1 \cdot 10$ & $\begin{array}{c}0.08 \\
<0.001\end{array}$ \\
\hline Median & \multicolumn{2}{|c|}{$4 \cdot 1$} & \multicolumn{2}{|c|}{$3 \cdot 2$} & \\
\hline 25th, 75th percentile & \multicolumn{2}{|c|}{$2 \cdot 9,5 \cdot 7$} & \multicolumn{2}{|c|}{$2 \cdot 2,4 \cdot 9$} & \\
\hline Weight status (\% uw/nw/ow/ob) & \multirow{2}{*}{\multicolumn{2}{|c|}{$\begin{array}{l}11 / 74 / 13 / 2 \\
2 / 36 / 18 / 44\end{array}$}} & \multicolumn{2}{|c|}{$9 / 78 / 11 / 2$} & 0.50 \\
\hline Month of blood sampling (\% August/September/October/November) & & & \multicolumn{2}{|c|}{$3 / 38 / 19 / 40$} & 0.58 \\
\hline Serum 25(OH)D (nmol/l) & $58 \cdot 4$ & $18 \cdot 1$ & $62 \cdot 9$ & $19 \cdot 0$ & $<0.001$ \\
\hline Whole-blood EPA + DHA (weight \%) & $3 \cdot 60$ & 0.98 & 3.58 & 0.95 & 0.68 \\
\hline PTH (pmol/l) & & & & & 0.02 \\
\hline Median & \multirow{2}{*}{\multicolumn{2}{|c|}{$\begin{array}{c}3 \cdot 2 \\
2 \cdot 4,4 \cdot 2\end{array}$}} & \multicolumn{2}{|c|}{$3 \cdot 1$} & \\
\hline 25th, 75th percentile & & & \multirow{2}{*}{\multicolumn{2}{|c|}{$2 \cdot 2,4 \cdot 0$}} & \\
\hline Moderate-to-vigorous physical activity (min/d) & & & & $<0.001$ \\
\hline Median & \multirow{2}{*}{\multicolumn{2}{|c|}{$\begin{array}{c}37 \\
26,48\end{array}$}} & \multirow{2}{*}{\multicolumn{2}{|c|}{$\begin{array}{c}57 \\
39,73\end{array}$}} & \\
\hline 25th, 75th percentile & & & & & \\
\hline \multicolumn{6}{|l|}{ Dietary intake } \\
\hline Energy intake $(\mathrm{kJ} / \mathrm{d})$ & 7210 & 1174 & 8290 & 1406 & $<0.001$ \\
\hline Fat (energy \%) & 31.9 & 4.0 & $32 \cdot 2$ & $4 \cdot 3$ & 0.32 \\
\hline Protein (energy \%) & $15 \cdot 1$ & $2 \cdot 1$ & $15 \cdot 5$ & $2 \cdot 0$ & 0.02 \\
\hline Carbohydrate (energy \%) & 53.0 & 4.6 & $52 \cdot 3$ & 4.7 & 0.06 \\
\hline $\mathrm{Ca}(\mathrm{mg} / \mathrm{d})$ & & & & & $<0.001$ \\
\hline Median & \multirow{2}{*}{\multicolumn{2}{|c|}{$\begin{array}{c}843 \\
684,1006\end{array}$}} & & \\
\hline 25th, 75th percentile & & & & & \\
\hline Vitamin D $(\mu \mathrm{g} / \mathrm{d})$ & & \multicolumn{2}{|c|}{796,1180} & $<0.01$ \\
\hline Median & & & & & \\
\hline 25th, 75th percentile & & & & & \\
\hline Vitamin D supplements (\% yes) $\dagger$ & & & & & 0.24 \\
\hline
\end{tabular}

25(OH)D, 25-hydroxy vitamin D; A, slower secondary education; B, upper secondary education; C, vocational education; D, short higher education; E, bachelor's degree or equivalent; $\mathrm{F}, \geq$ master's degree; $\mathrm{nw}$, normal weight; ob, obese; ow, overweight; PTH, parathyroid hormone; uw, underweight.

* $n$ 756-782; however $n 727$ for PTH and $n 681$ for dietary intake.

$\dagger$ Registered intake of vitamin D-containing dietary supplements $\geq 1 \mathrm{~d}$ during the dietary recording week.

Table 2. Cardiometabolic risk markers in the study population

(Mean values and standard deviations; median values and 25th, 75th percentiles)

\begin{tabular}{|c|c|c|c|c|c|c|}
\hline & \multirow[b]{2}{*}{$n$} & \multicolumn{2}{|c|}{ Girls } & \multicolumn{2}{|c|}{ Boys } & \multirow[b]{2}{*}{$P$} \\
\hline & & Mean & SD & Mean & SD & \\
\hline HOMA-IR & 721 & & & & & 0.002 \\
\hline $\begin{array}{l}\text { Median } \\
\text { 25th, 75th percentile }\end{array}$ & & \multicolumn{2}{|c|}{$\begin{array}{c}1 \cdot 53 \\
1 \cdot 10,2 \cdot 13\end{array}$} & \multicolumn{2}{|c|}{$\begin{array}{c}1.35 \\
0.99,1.91\end{array}$} & \\
\hline Systolic blood pressure $(\mathrm{mmHg})$ & 782 & 107 & 9 & 108 & 8 & 0.08 \\
\hline Diastolic blood pressure $(\mathrm{mmHg})$ & 782 & 69 & 7 & 67 & 6 & $<0.001$ \\
\hline Total cholesterol $(\mathrm{mmol} / \mathrm{l})$ & 782 & $4 \cdot 11$ & 0.65 & 4.08 & 0.66 & 0.54 \\
\hline LDL-cholesterol (mmol/l/) & 782 & 2.37 & 0.56 & $2 \cdot 30$ & 0.58 & 0.09 \\
\hline HDL-cholesterol (mmol/li) & 782 & 1.40 & 0.29 & 1.49 & 0.32 & $<0.001$ \\
\hline TAG $(\mathrm{mmol} / \mathrm{l})$ & 782 & & & & & $<0.001$ \\
\hline Median & & \multirow{2}{*}{\multicolumn{2}{|c|}{$\begin{array}{c}0.66 \\
0.54,0.84\end{array}$}} & \multirow{2}{*}{\multicolumn{2}{|c|}{$\begin{array}{c}0.58 \\
0.48,0.71\end{array}$}} & \\
\hline 25th, 75th percentile & & & & & & \\
\hline Waist circumference $(\mathrm{cm})$ & 782 & & & & & 0.95 \\
\hline Median & & \multirow{2}{*}{\multicolumn{2}{|c|}{$\begin{array}{c}62 \cdot 7 \\
59 \cdot 0,68 \cdot 7\end{array}$}} & \multirow{2}{*}{\multicolumn{2}{|c|}{$\begin{array}{c}62 \cdot 6 \\
59 \cdot 4.68 \cdot 3\end{array}$}} & \\
\hline 25th, 75th percentile & & & & & & \\
\hline Plasma adiponectin (mg/l) & 759 & & & & & 0.08 \\
\hline Median & & \multirow{2}{*}{\multicolumn{2}{|c|}{$\begin{array}{c}11 \cdot 3 \\
8 \cdot 3,14 \cdot 9\end{array}$}} & \multirow{2}{*}{\multicolumn{2}{|c|}{$\begin{array}{c}10 \cdot 6 \\
7 \cdot 7,14 \cdot 1\end{array}$}} & \\
\hline 25th, 75th percentile & & & & & & \\
\hline Plasma IL-6 (ng/l) & 774 & & & & & $<0.001$ \\
\hline Median & & \multirow{2}{*}{\multicolumn{2}{|c|}{$\begin{array}{c}0.95 \\
0.69,1.56\end{array}$}} & \multirow{2}{*}{\multicolumn{2}{|c|}{$\begin{array}{c}0.80 \\
0.58,1.18\end{array}$}} & \\
\hline 25th, 75th percentile & & & & & & \\
\hline MetS score* & 744 & 0.55 & 3.33 & -0.48 & 2.95 & $<0.001$ \\
\hline
\end{tabular}

HOMA-IR, homoeostasis model of assessment-insulin resistance; MetS: metabolic syndrome.

* Based on mean arterial pressure, HDL-cholesterol, TAG, HOMA-IR and waist circumference (see the 'Methods' section). 
Table 3. Associations between serum 25-hydroxy vitamin D (25(OH)D) and cardiometabolic risk markers ( $\beta$ Coefficients and $95 \%$ confidence intervals)

\begin{tabular}{|c|c|c|c|c|c|c|c|c|c|}
\hline & \multicolumn{3}{|c|}{$25(\mathrm{OH}) \mathrm{D}$} & \multicolumn{3}{|c|}{ 25(OH)D adjusted for FMI } & \multicolumn{3}{|c|}{ 25(OH)D adjusted for MVPA } \\
\hline & $\beta$ & $95 \% \mathrm{Cl}^{*}$ & $P$ & $\beta$ & $95 \% \mathrm{Cl}^{*}$ & $P$ & $\beta$ & $95 \% \mathrm{Cl}^{*}$ & $P$ \\
\hline HOMA-IR & -0.02 & $-0.06,0.00$ & 0.12 & - & & - & - & & - \\
\hline Systolic blood pressure (mmHg) & $-0 \cdot 2$ & $-0.6,0.1$ & 0.22 & - & & - & - & & - \\
\hline Diastolic blood pressure $(\mathrm{mmHg})$ & -0.3 & $-0.6,-0.0$ & 0.02 & -0.3 & $-0.6,-0.0$ & 0.04 & -0.3 & $-0.5,0.0$ & 0.06 \\
\hline Total cholesterol $(\mathrm{mmol} / \mathrm{l})$ & -0.07 & $-0.10,-0.05$ & $<0.001$ & -0.07 & $-0.10,-0.04$ & $<0.001$ & -0.08 & $-0.10,-0.05$ & $<0.001$ \\
\hline LDL-cholesterol (mmol/l) & -0.05 & $-0.08,-0.03$ & $<0.001$ & -0.05 & $-0.07,-0.02$ & $<0.001$ & -0.06 & $-0.08,-0.03$ & $<0.001$ \\
\hline HDL-cholesterol (mmol/l) & -0.01 & $-0.02,0.00$ & 0.15 & - & & - & - & & - \\
\hline TAG (mmol/l) & -0.02 & $-0.03,-0.01$ & 0.001 & -0.01 & $-0.02,-0.01$ & 0.003 & -0.02 & $-0.03,-0.01$ & 0.001 \\
\hline Waist circumference $(\mathrm{cm}) \dagger$ & -0.24 & $-0.51,0.03$ & 0.09 & - & & - & - & & - \\
\hline MetS score†‡ & -0.16 & $-0.28,-0.03$ & 0.01 & - & - & - & $-0 \cdot 13$ & $-0.25,0.00$ & 0.047 \\
\hline Adiponectin (mg/l) & 102 & $-124,328$ & 0.38 & - & & - & - & & - \\
\hline IL-6 (ng/l) & 0.01 & $-0.02,0.04$ & 0.49 & - & & - & - & & - \\
\hline
\end{tabular}

FMI, fat mass index; HOMA-IR, homoeostasis model of assessment-insulin resistance; MetS, metabolic syndrome; MVPA, moderate-to-vigorous physical activity (min/d).

* Mixed linear models, all adjusted for sex, age, height, ethnicity, whole-blood EPA + DHA, entered puberty (yes/no) and parental education. Regression coefficients are presented as cardiometabolic outcome response per $10 \mathrm{nmol} / \mathrm{l}$ increase in serum 25(OH)D.

$\dagger$ Because of high risk of colinearity, waist circumference and the MetS score were not adjusted for FMI.

‡ Based on mean arterial pressure, plasma HDL-cholesterol, plasma TAG, HOMA-IR and waist circumference.

pressure became borderline significant after adjustment for MVPA. Adjustment for BMI $Z$-scores instead of FMI or for PTH did not change the results (data not shown).

Only fifteen children (1.9\%) had plasma TAG $\geq 1.7 \mathrm{mmol} / \mathrm{l}$. The same number had high blood pressure defined as $\geq 130$ / $85 \mathrm{mmHg}$, whereas thirty-seven children (4.7\%) had HDLcholesterol values $<1.03 \mathrm{mmol} / \mathrm{l}$. A total of sixty-five children (9.0\%) had HOMA-IR $>2.8$ and 265 children (33.9\%) had a waist circumference $\geq 90$ th percentile ${ }^{(47)}$. Elevated total and LDL-cholesterol $(\geq 5.2$ and $\geq 3.4 \mathrm{mmol} / 1$, respectively) were found in fifty $(6.4 \%)$ and thirty-nine $(5.0 \%)$ children, respectively. Higher serum $25(\mathrm{OH}) \mathrm{D}$ was associated with reduced odds for high total cholesterol - that is, OR 0.80 (95\% CI 0.66, 0.97) $(P=0.03)$ per $10 \mathrm{nmol} / \mathrm{l}$ increase in serum 25(OH)D. This was not seen for LDL-cholesterol $(P=0.93)$ or any of the other cardiometabolic markers $(P>0 \cdot 12)$.

\section{Discussion}

This study showed inverse associations between serum vitamin D status and a number of cardiometabolic risk markers in a sample of primarily normal weight, healthy children with low prevalence of vitamin D deficiency. To our surprise, the associations with plasma cholesterols, TAG and the continuous MetS score were independent of the potential confounding effects of fat mass and physical activity. The negative association with the MetS score was likely driven by TAG and to some degree by waist circumference, as total and LDL-cholesterol were not included in the score.

Some previous studies among child populations of fairly comparable ethnicity, age and body size have also demonstrated inverse associations between vitamin $\mathrm{D}$ status and total cholesterol $^{(20,50)}$, TAG $^{(50,51)}$, diastolic blood pressure ${ }^{(20,52)}$ and waist circumference ${ }^{(16)}$, whereas four studies in Canadian, Spanish, American and British children, respectively, found no associations with LDL-cholesterol ${ }^{(24,50-52)}$. A study in 149 Spanish children reported an inverse association with TAG, but not with total cholesterol, after adjustment for age, sex, BMI and physical activity ${ }^{(51)}$. Whereas associations with HDL-cholesterol were not found in the present study, positive associations between serum 25(OH)D and HDL-cholesterol were reported in large samples of American ${ }^{(20)}$ and British children $^{(52)}$. In the British study, the association with HDL-cholesterol, but not that with blood pressure, remained after adjustment for waist circumference, socioeconomic position and other potential confounders ${ }^{(52)}$. None of the studies investigating associations with plasma lipid profile adjusted for puberty, which may be an important confounder.

In the present study, vitamin D status was positively associated with BMI and FMI. However, only the association with FMI remained after adjustment for parental education. This indicates that socioeconomic differences may indeed be confounding the association with BMI, probably through an effect on behaviours related to both vitamin D exposure and being overweight. However, in line with the persisting association with FMI in the present study, a randomised controlled trial in African-American children reported that the serum $25(\mathrm{OH}) \mathrm{D}$ response to vitamin D supplementation was consistently inversely correlated with baseline total body fat mass ${ }^{(53)}$. Similarly, an American vitamin D supplementation trial in Caucasian adolescents found increments in $25(\mathrm{OH}) \mathrm{D}$ concentrations to be lower in obese compared with non-obese adolescents $^{(54)}$, indicating that vitamin $\mathrm{D}$ sequestering in adipose tissue may indeed have a role.

We showed that associations between vitamin D status and blood pressure and plasma lipids were independent of fat mass and BMI, indicating potential independent mechanisms. It has been demonstrated that the active metabolite of vitamin $\mathrm{D}$, $1,25(\mathrm{OH})_{2} \mathrm{D}$, can upregulate lipoprotein lipase in vitro, thereby potentially playing a beneficial role in lipoprotein metabolism ${ }^{(55)}$. However, causality cannot be inferred from the present study, and to our knowledge no randomised controlled trials have investigated the effect of vitamin D supplementation on plasma lipids in children. A non-randomised study 
supplementing seventy Argentine Indian school children with $5000 \mathrm{IU}(125 \mu \mathrm{g} / \mathrm{d})$ vitamin D for 8 weeks showed that HDLcholesterol measured after 1 year was increased compared with a group of twenty unsupplemented children ${ }^{(56)}$. In contrast with the present study, the study population had high prevalence of vitamin D deficiency - that is, $20 \%$ of the children had serum $25(\mathrm{OH}) \mathrm{D}<25 \mathrm{nmol} / \mathrm{l}$. With regard to blood pressure, there is some evidence, especially from vitamin D receptor knockout mice, that vitamin $\mathrm{D}$ can reduce blood pressure through inhibition of the renin-angiotensin system ${ }^{(6)}$. Other suggested mechanisms include regulation of vascular tone by $1,25(\mathrm{OH}) \mathrm{D}$ by facilitating $\mathrm{Ca}$ influx to the muscle cells and direct effects on vascular endothelium and smooth muscle cells ${ }^{(57)}$ - for example, through regulation of transcription of endothelial nitric oxide synthase, which has been demonstrated in vitro ${ }^{(58)}$. However, the association between vitamin D status and blood pressure was somewhat confounded by physical activity in the present study. This indicates that behaviours related to both vitamin $\mathrm{D}$ exposure and blood pressure rather than biological mechanisms may be responsible for the association. To our knowledge, only the single-blinded randomised controlled trial in forty-nine African-American adolescents has investigated the effects of vitamin D on blood pressure. The participants, who had a mean serum $25(\mathrm{OH}) \mathrm{D}$ of $33.4 \mathrm{nmol} / \mathrm{l}$ at baseline, were randomised to $2000 \mathrm{IU} / \mathrm{d}(50 \mu \mathrm{g} / \mathrm{d})$ or $400 \mathrm{IU} / \mathrm{d}(10 \mu \mathrm{g} / \mathrm{d})$ (control) vitamin $\mathrm{D}$ for 16 weeks from February to May. Although carotid-femoral pulse-wave velocity increased in the control group and decreased in the high-dose vitamin D group, no changes or differences were seen in systolic or diastolic blood pressure ${ }^{(53)}$. Meta-analyses of randomised trials in adults show no or little effect of vitamin D on blood pressure. Witham et $a l .{ }^{(59)}$ included eleven randomised controlled vitamin D trials of mainly older subjects and found a small reduction in diastolic blood pressure $(-3.1 \mathrm{mmHg}, 95 \% \mathrm{CI}-5.5,-0.6)$ among the eight studies in hypertensive subjects only. However, as most studies were relatively small (less than sixty subjects) and significant heterogeneity was present for the subgroup analyses, the reliability of these findings is unclear. This area calls for further investigation, especially in children.

MVPA was also positively associated with vitamin D status in the present study, something that has previously been observed in Korean school children of similar age ${ }^{(27)}$. In the present study, this level of physical activity could be hypothesised to be a proxy for sun exposure because of the outdoor nature of much MVPA during the period from August to November where data were collected. Indeed, positive associations have been observed between outdoor engagement and physical activity in the OPUS School Meal Study ${ }^{(35)}$ and among other children of this age ${ }^{(60,61)}$. Physical activity could also be a proxy for a particular lifestyle and an eating pattern with higher intake of fish (the main food source of vitamin D in Danes), as has been demonstrated in previous studies among children ${ }^{(62,63)}$. In addition, we have recently shown that $n$ - 3 long-chain PUFA in blood, a biomarker of fish intake, was cross-sectionally associated with cardiometabolic risk markers in the OPUS School Meal Study ${ }^{(45)}$. However, the presented results were adjusted for this biomarker. Finally, although we found a strong inverse association between PTH and vitamin D status in the present study, the observed associations between vitamin D status and the cardiometabolic markers did not appear to be mediated by PTH.

To our knowledge, this is the first study of its kind among children from the Nordic countries. Vitamin D status was measured solely during autumn and early winter months, a time of year when vitamin D status is expected to be close to annual peak in Danish children. Still, about every fourth child had serum $25(\mathrm{OH}) \mathrm{D}$ status $\leq 50 \mathrm{nmol} / \mathrm{l}$. A limitation to autumn $25(\mathrm{OH}) \mathrm{D}$ measurements is that they are likely influenced by recent sun behaviour including sunny vacations abroad, which were not recorded in the study. However, a recent study in Danish girls showed that the $25(\mathrm{OH}) \mathrm{D}$ concentrations attained during summer were strong predictors of vitamin D status the following winter ${ }^{(64)}$. The explorative nature of the study requested multiple testing and the results must naturally be interpreted with this in mind. In addition, although we were able to adjust for important potential confounders such as pubertal status, ethnicity and the biomarker of fish intake, there is always a risk of residual confounding in cross-sectional studies such as the present one. However, the robust findings throughout the adjustments and the consistently low $P$ values for the plasma lipids do not suggest random findings. It is a great strength that the study included DXA scans and $7 \mathrm{~d}$ accelerometry measurements, as these provided validated and objective measurements of body fat and physical activity. The observed associations were of small magnitude, and although higher serum $25(\mathrm{OH}) \mathrm{D}$ was associated with reduced odds for high total cholesterol values this was not the case for HDL-cholesterol or TAG. Therefore, the immediate clinical relevance is uncertain. However, plasma lipid levels and blood pressure have been shown to track from childhood to adulthood $^{(65)}$ and children with the MetS in childhood are more likely to have the MetS as adults ${ }^{(66)}$. This indicates that from a population perspective slightly lower levels of the cardiometabolic risk markers may give long-term benefit, if sustained over years. In line with this, higher serum 25(OH)D seemed to be associated with more beneficial HDL-cholesterol, TAG and MetS scores over the entire range of values in the present study, rather than having a specific impact on the proportion of aberrant values. There is no uniformly used set of cut-offs for cardiometabolic risk markers in children, and the various cut-offs for aberrant values should be used and interpreted with caution. The very high proportion of children with waist circumference above the 90th percentile in the present study, despite the use of a recently published Danish reference material ${ }^{(47)}$, is hard to explain, but it may relate to the fact that we measured waist circumference at the level of the umbilicus, whereas the reference study measured parallel to the floor at the midpoint between the ribs and the top of the iliac crest.

In conclusion, vitamin D status was negatively associated with a number of cardiometabolic markers in Danish school children with low prevalence of vitamin D deficiency, and apart from blood pressure the associations were independent of body fat and physical activity. The potential underlying cause-effect relationship and possible long-term health implications should be investigated further in randomised controlled trials. 


\section{Acknowledgements}

The authors thank the participating children, their families, the school managements, teachers and other staff, as well as the entire study team, for their contribution to the study.

The Optimal well-being, development and health for Danish children through a healthy New Nordic Diet (OPUS) study was supported by the Nordea Foundation (grant no: 02-2010-0389). The funder had no role in the design and analysis of the study or in the writing of this article.

R. A. P., C. M., K. F. M., A. A., I. T., L. B. S., S. M. D. and C. T. D. designed the research; R. A., M. F. H., L. B. S., S. M. D., C. T. D. and R. A. P. conducted the research; R. A. P. and C. R. analysed the data; H. K. provided acquisition of the data; R. A. P. and C. T. D. wrote the paper; and R. A. P. and C. T. D. had primary responsibility for the final content.

R. A. P., S. M. D., L. B. S., M. F. H., R. A., I. T., H. K., C. R., K. F. M., C. M. and C. T. D. declare no conflicts of interest that could be perceived as prejudicing the impartiality of the research reported. A. A. is an ad hoc consultant for clients of Gerson Lehrman Group (USA) and consultant/member of advisory/ scientific boards for Global Dairy Platform (USA), Kraft Foods (USA), Dutch Beer Knowledge Institute (The Netherlands), McCain Foods Limited (USA), McDonald's (USA) and Jenny Craig (USA) and receives honoraria as speaker and research funding from a range of Danish and international companies.

\section{References}

1. Holick MF (2004) Sunlight and vitamin D for bone health and prevention of autoimmune diseases, cancers, and cardiovascular disease. Am J Clin Nutr 80, 1678S-1688S.

2. Bouillon R, Van Cromphaut S \& Carmeliet G (2003) Intestinal calcium absorption: molecular vitamin D mediated mechanisms. J Cell Biochem 88, 332-339.

3. Christakos S (2011) Mechanism of action of 1,25dihydroxyvitamin $\mathrm{D}(3)$ on intestinal calcium absorption. Rev Endocr Metab Disord 347, 25-29.

4. Wharton B \& Bishop N (2003) Rickets. Lancet 362, 1389-1400.

5. Dolinsky DH, Armstrong S, Mangarelli C, et al. (2013) The association between vitamin D and cardiometabolic risk factors in children: a systematic review. Clin Pediatr 52, 210-223.

6. Li YC (2003) Vitamin D regulation of the reninangiotensin system. $J$ Cell Biochem 88, 327-331.

7. Wolden-Kirk H, Overbergh L, Christesen HT, et al. (2011) Vitamin D and diabetes: its importance for beta cell and immune function. Mol Cell Endocrinol 347, 106-120.

8. Wolden-Kirk H, Rondas D, Bugliani M, et al. (2014) Discovery of molecular pathways mediating 1,25-dihydroxyvitamin D protection against cytokine-induced inflammation and damage of human and male mouse islets of Langerhans. Endocrinology 155, 736-747.

9. Querfeld U (2013) Vitamin D and inflammation. Pediatr Nephrol 28, 605-610.

10. Marcotorchino J, Gouranton E, Romier B, et al. (2012) Vitamin $\mathrm{D}$ reduces the inflammatory response and restores glucose uptake in adipocytes. Mol Nutr Food Res 56, 1771-1782.

11. Alemzadeh R \& Kichler J (2012) Parathyroid hormone is associated with biomarkers of insulin resistance and inflammation, independent of vitamin D status, in obese adolescents. Metab Syndr Relat Disord 10, 422-429.
12. Gilardini L, Pasqualinotto L, Di Matteo S, et al. (2011) Factors associated with early atherosclerosis and arterial calcifications in young subjects with a benign phenotype of obesity. Obesity 19, 1684-1689.

13. Zimmet $\mathrm{P}$, Alberti KG, Kaufman F, et al. (2007) The metabolic syndrome in children and adolescents - an IDF consensus report. Pediatr Diabetes 8, 299-306.

14. Nadeau KJ, Maahs DM, Daniels SR, et al. (2011) Childhood obesity and cardiovascular disease: links and prevention strategies. Nat Rev Cardiol 8, 513-525.

15. Saneei P, Salehi-Abargouei A \& Esmaillzadeh A (2013) Serum 25-hydroxy vitamin D levels in relation to body mass index: a systematic review and meta-analysis. Obes Rev 14, 393-404.

16. Pacifico L, Anania C, Osborn JF, et al. (2011) Low 25(OH)D3 levels are associated with total adiposity, metabolic syndrome, and hypertension in Caucasian children and adolescents. Eur J Endocrinol 165, 603-611.

17. Lagunova Z, Porojnicu AC, Lindberg FA, et al. (2011) Vitamin D status in Norwegian children and adolescents with excess body weight. Pediatric diabetes 12, 120-126.

18. Alemzadeh R, Kichler J, Babar G, et al. (2008) Hypovitaminosis $\mathrm{D}$ in obese children and adolescents: relationship with adiposity, insulin sensitivity, ethnicity, and season. Metabolism 57, 183-191.

19. Olson ML, Maalouf NM, Oden JD, et al. (2012) Vitamin D deficiency in obese children and its relationship to glucose homeostasis. J Clin Endocrinol Metab 97, 279-285.

20. Kumar J, Muntner P, Kaskel FJ, et al. (2009) Prevalence and associations of 25-hydroxyvitamin D deficiency in US children: NHANES 2001-2004. Pediatrics 124, e362-e370.

21. Rodriguez-Rodriguez E, Navia-Lomban B, Lopez-Sobaler AM, et al. (2010) Associations between abdominal fat and body mass index on vitamin D status in a group of Spanish schoolchildren. Eur J Clin Nutr 64, 461-467.

22. Codoner-Franch $\mathrm{P}$, Tavarez-Alonso S, Simo-Jorda $\mathrm{R}$, et al. (2012) Vitamin D status is linked to biomarkers of oxidative stress, inflammation, and endothelial activation in obese children. J Pediatr 161, 848-854.

23. Aypak C, Turedi O \& Yuce A (2013) The association of vitamin D status with cardiometabolic risk factors, obesity and puberty in children. Eur J Pediatr 173, 367-373.

24. Sacheck J, Goodman E, Chui K, et al. (2011) Vitamin D deficiency, adiposity, and cardiometabolic risk in urban schoolchildren. J Pediatr 159, 945-950.

25. Earthman CP, Beckman LM, Masodkar K, et al. (2012) The link between obesity and low circulating 25-hydroxyvitamin D concentrations: considerations and implications. Int J Obes $\mathbf{3 6}$, 387-396.

26. Ekelund U, Luan J, Sherar LB, et al. (2012) Moderate to vigorous physical activity and sedentary time and cardiometabolic risk factors in children and adolescents. JAMA 307, 704-712.

27. Ha CD, Cho JK, Lee SH, et al. (2013) Serum vitamin D, physical activity, and metabolic risk factors in Korean children. Med Sci Sports Exerc 45, 102-108.

28. Damsgaard CT, Dalskov SM, Petersen RA, et al. (2012) Design of the OPUS School Meal Study: a randomised controlled trial assessing the impact of serving school meals based on the New Nordic Diet. Scand J Public Health 40, 693-703.

29. Statistics Denmark (2012) Indvandrere i Danmark (Immigrants in Denmark). Copenhagen: Rosendahls - Schultz Grafisk A/S. http://www.dst.dk/pukora/epub/upload/16601/indv.pdf (accessed July 2015).

30. Morris N \& Udry JR (1980) Validation of a self-administered instrument to assess stage of adolescent development. J Youth Adolesc 9, 271-280. 
31. Biltoft-Jensen A, Trolle E, Christensen T, et al. (2014) WebDASC: a web-based dietary assessment software for 8-11-year-old Danish children. J Hum Nutr Diet 27, Suppl. 1, 43-53.

32. Biltoft-Jensen A, Hjorth MF, Trolle E, et al. (2013) Comparison of estimated energy intake using web-based dietary assessment software with accelerometer-determined energy expenditure in children. Food Nutr Res 57 (epublication 17 December 2013).

33. Henry CJ (2005) Basal metabolic rate studies in humans: measurement and development of new equations. Public Health Nutr 8, 1133-1152.

34. Black AE (2000) The sensitivity and specificity of the Goldberg cut-off for EI:BMR for identifying diet reports of poor validity. Eur J Clin Nutr 54, 395-404

35. Hjorth MF, Chaput JP, Michaelsen K, et al. (2013) Seasonal variation in objectively measured physical activity, sedentary time, cardio-respiratory fitness and sleep duration among 8-11 year-old Danish children: a repeated-measures study. BMC Public Health 13, 808.

36. Trost SG, Loprinzi PD, Moore R, et al. (2011) Comparison of accelerometer cut points for predicting activity intensity in youth. Med Sci Sports Exerc 43, 1360-1368.

37. Damsgaard CT, Dalskov SM, Laursen RP, et al. (2014) Provision of healthy school meals does not affect the metabolic syndrome score in 8-11-year-old children, but reduces cardiometabolic risk markers despite increasing waist circumference. Br J Nutr 112, 1826-1836.

38. World Health Organization (2012) WHO anthroplus macros for STATA. http://www.who.int/growthref/tools/en/ (accessed September 2013)

39. Cole TJ, Flegal KM, Nicholls D, et al. (2007) Body mass index cut offs to define thinness in children and adolescents: international survey. BMJ 335, 194.

40. Cole TJ, Bellizzi MC, Flegal KM, et al. (2000) Establishing a standard definition for child overweight and obesity worldwide: international survey. BMJ 320, 1240-1243.

41. Friedewald WT, Levy RI \& Fredrickson DS (1972) Estimation of the concentration of low-density lipoprotein cholesterol in plasma, without use of the preparative ultracentrifuge. Clin Chem 18, 6, 499-502.

42. Cutfield WS, Jefferies CA, Jackson WE, et al. (2003) Evaluation of HOMA and QUICKI as measures of insulin sensitivity in prepubertal children. Pediatr Diabetes 4, 119-125.

43. Carter GD, Berry JL, Gunter E, et al. (2010) Proficiency testing of 25-hydroxyvitamin D (25-OHD) assays. J Steroid Biochem Mol Biol 121, 176-179.

44. Braegger C, Campoy C, Colomb V, et al. (2013) Vitamin D in the Healthy European Paediatric Population. I Pediatr Gastroenterol Nutr 56, 692-701.

45. Damsgaard CT, Eidner MB, Stark KD, et al. (2014) Eicosapentaenoic acid and docosahexaenoic acid in whole blood are differentially and sex-specifically associated with cardiometabolic risk markers in 8-11-year-old Danish children. PLOS ONE 9, e109368.

46. Gobel RJ, Jensen SM, Frokiaer H, et al. (2012) Obesity, inflammation and metabolic syndrome in Danish adolescents. Acta Paediatr 101, 192-200.

47. Wohlfahrt-Veje C, Tinggaard J, Winther K, et al. (2014) Body fat throughout childhood in 2647 healthy Danish children: agreement of BMI, waist circumference, skinfolds with dual X-ray absorptiometry. Eur J Clin Nutr 68, 664-670.

48. Tresaco B, Bueno G, Pineda I, et al. (2005) Homeostatic model assessment (HOMA) index cut-off values to identify the metabolic syndrome in children. J Physiol Biochem 61,381-388.

49. National Heart, Lung, and Blood Institute (2012) Expert Panel on Integrated Guidelines for Cardiovascular Health and Risk Reduction in Children and Adolescents Full Report no. NIH
Publication No. 12-7486. Bethesda, MD: US Department of Health and Human Services, National Institutes of Health.

50. Delvin EE, Lambert M, Levy E, et al. (2010) Vitamin D status is modestly associated with glycemia and indicators of lipid metabolism in French-Canadian children and adolescents. J Nutr 140, 987-991.

51. Rodriguez-Rodriguez E, Ortega RM, Gonzalez-Rodriguez LG, et al. (2011) Vitamin D deficiency is an independent predictor of elevated triglycerides in Spanish school children. Eur J Nutr 50, 373-378.

52. Williams DM, Fraser A, Sayers A, et al. (2012) Associations of 25-hydroxyvitamin $D_{2}$ and $D_{3}$ with cardiovascular risk factors in childhood: cross-sectional findings from the Avon Longitudinal Study of Parents and Children. I Clin Endocrinol Metab 97, 1563-1571.

53. Dong Y, Stallmann-Jorgensen IS, Pollock NK, et al. (2010) A 16-week randomized clinical trial of 2000 international units daily vitamin $\mathrm{D}_{3}$ supplementation in black youth: 25-hydroxyvitamin D, adiposity, and arterial stiffness. J Clin Endocrinol Metab 95, 4584-4591.

54. Aguirre Castaneda R, Nader N, Weaver A, et al. (2012) Response to vitamin $\mathrm{D}_{3}$ supplementation in obese and non-obese Caucasian adolescents. Horm Res Paediatr 78, 226-231.

55. Querfeld U, Hoffmann MM, Klaus G, et al. (1999) Antagonistic effects of vitamin D and parathyroid hormone on lipoprotein lipase in cultured adipocytes. J Am Soc Nephrol 10, 2158-2164.

56. Hirschler V, Maccallini G, Sanchez MS, et al. (2013) Improvement in high-density lipoprotein cholesterol levels in argentine Indian school children after vitamin D supplementation. Horm Res Paediatr 80, 335-342.

57. Vaidya A \& Forman JP (2010) Vitamin D and hypertension: current evidence and future directions. Hypertension 56, 774-779.

58. Talmor Y, Golan E, Benchetrit S, et al. (2008) Calcitriol blunts the deleterious impact of advanced glycation end products on endothelial cells. Am J Physiol Renal Physiol 294, F1059-F1064.

59. Witham MD, Nadir MA \& Struthers AD (2009) Effect of vitamin D on blood pressure: a systematic review and meta-analysis. J Hypertens 27, 1948-1954.

60. Nielsen G, Taylor R, Williams S, et al. (2010) Permanent play facilities in school playgrounds as a determinant of children's activity. J Phys Act Health 7, 490-496.

61. Nilsson A, Andersen LB, Ommundsen Y, et al. (2009) Correlates of objectively assessed physical activity and sedentary time in children: a cross-sectional study (The European Youth Heart Study). BMC Public Health 9, 322.

62. Morin P, Turcotte S \& Perreault G (2013) Relationship between eating behaviors and physical activity among primary and secondary school students: results of a crosssectional study. J Sch Health 83, 597-604.

63. Ottevaere C, Huybrechts I, Beghin L, et al. (2011) Relationship between self-reported dietary intake and physical activity levels among adolescents: the HELENA study. I Int J Behav Nutr Phys Act 8, 8.

64. Andersen R, Brot C, Jakobsen J, et al. (2013) Seasonal changes in vitamin D status among Danish adolescent girls and elderly women: the influence of sun exposure and vitamin D intake. Eur J Clin Nutr 67, 270-274.

65. Juhola J, Magnussen CG, Viikari JS, et al. (2011) Tracking of serum lipid levels, blood pressure, and body mass index from childhood to adulthood: the Cardiovascular Risk in Young Finns Study. J Pediatr 159, 584-590.

66. Morrison JA, Friedman LA, Wang P, et al. (2008) Metabolic syndrome in childhood predicts adult metabolic syndrome and type 2 diabetes mellitus 25 to 30 years later. J Pediatr $\mathbf{1 5 2}$, 201-206. 